\title{
Human Infected H5N1 Avian Influenza
}

\author{
Puxuan Lu, Boping Zhou, Jing Yuan, and Guilin Yang
}

\subsection{Introduction}

\subsubsection{Definition and Prevalence}

Avian influenza is an infectious disease caused by avian influenza virus, which is also known as avian plague or European avian plague.

The influenza virus that infects poultry is known as avian influenza virus (AIV), which is categorized into the genus of influenza virus $\mathrm{A}$ and the family of Orthomyxoviridae. Currently, AVI is categorized into $16 \mathrm{H}$ subtypes $(\mathrm{H} 1 \sim \mathrm{H} 16)$ and $9 \mathrm{~N}$ subtypes (N1 N9) according to the respective antigenicity of hemagglutinin (HA) and neuramidinase (NA). In addition to poultry, AVI can also infect I human, pig, horse, mink and marine mammals. According to the varying virulence of AVI to chicken, it is graded into highly pathogenic, moderately pathogenic and mildly or non-pathogenic.

Human infected $\mathrm{H} 5 \mathrm{~N} 1$ avian influenza is an acute respiratory infectious disease caused by avian influenza virus (H5N1). It is also known as highly pathogenic avian influenza (HPAI) due to its serious symptoms and high mortality. The 1 case of human infected H5N1 avian influenza, a 3-year-old child, was reported in Hong Kong on May 5, 1997. The child was hospitalized due to cold and fever but incurable by therapies, and death occurred due to Reye syndrome and multiple organs failure 10 days after the onset. On May 9, 1997, a strain of H5N1 AIV, nominated as A/ Hongkong/157/97, was isolated from his tracheal secretion in National Central Laboratory of Influenza in Rotterdam, Holland and Center for Disease Control, the United States. Therefore, the child was etiologically diagnosed with human infected H5N1 avian influenza. And the child was the first case of human infected highly pathogenic avian influenza (H5N1). Within the following 6 months, 17 other cases were

P. Lu $(\bowtie) \cdot$ B. Zhou • J. Yuan • G. Yang Affiliated Shenzhen Third People's Hospital, Guangdong Medical College, Shenzhen, Guangdong, China

e-mail: lupuxuan@126.com reported, and the patients aged 1-60 years, with the same H5N1 virus strain isolated from their respiratory secretions. In December, 1997, AIV H5N1 was isolated in samples collected from a market selling alive poultry. By killing sick poultry in a large-scale, human infection was then rapidly controlled. In the year of 1997, a total of 18 cases human infected H5N1 avian influenza were reported, with 5 cases of death.

Since the first occurrence of human infected H5N1 avian influenza in HongKong in 1997, a total of 667 cases have been definitively diagnosed globally, with 393 cases of death by Dec. 31, 2014. And the death rate was $58.92 \%$. WHO warned that human infected H5N1 avian influenza may be one of the most potentially life-threatening diseases and should be highly concerned across the world.

\subsubsection{Epidemiology}

\subsubsection{Source of Infection}

The infected poultry by AIV H5N1 or the poultry carrying AIV H5N1, such as chicken, duck, goose, and dove, particularly chicken, are important sources of its infection. Wild aquatic birds (mostly with in apparent infection) play an important role in natural spread of the disease. Birds, such as swallow, chukar, bar-headed goose, crow, sparrow, and heron, may also act as the source of its infection. And migration of migrant birds also contributes to the transmission of AIV. Evidence is still needed to prove whether human is also a source of its infection.

\subsubsection{Route of Transmission}

\section{Respiratory Transmission}

Route of respiratory transmission refers to inhalation of droplets and droplet nuclei carrying pathogenic AIV particles. AIV infection spreads via airborne transmission, and human can be infected after direct contact to sick poultry or asymptomatic infected poultry, their secretions 
and excretions. Otherwise, human can also be infected after exposure to the contaminated environment by AIV. After such direct contact or exposure, the virus particles are inhaled into and attached to human upper respiratory tract for further invasion.

\section{Contact Transmission}

Transmission via direct or indirect contact refers to human infection of $\mathrm{H} 5 \mathrm{~N} 1$ avian influenza after close contact to feces of poultry/animal infected by AIV H5N1. Via direct or indirect contact, the virus can be inoculated into human upper respiratory tract, conjunctival mucosa or skin wound by itself.

\section{Susceptible Population}

The data from the cases with human infected H5N1 avian influenza indicated that human infected $\mathrm{H} 5 \mathrm{~N} 1$ avian influenza affects any age group and children under the age of 12 years shows a higher incidence with no gender difference. In addition, the patients from such an age group commonly experience serious conditions.

In addition, human infected $\mathrm{H} 5 \mathrm{~N} 1$ avian influenza can also spread via other routes of transmission, including gastrointestinal tract, skin wound, conjunctiva, aerosol, and blood. It may also spread vertically or in laboratory, traffic vehicle, and hospital.

\subsubsection{Clinical Manifestation}

In the early stage of human infected H5N1 avian influenza, the patients commonly experience common influenza like symptoms, including fever with a body temperature persistently above $39{ }^{\circ} \mathrm{C}$ with accompanying runny nose, nasal obstruction, cough, sore throat, headache, muscular soreness and general malaise. And some patients may experience gastrointestinal symptoms including nausea, abdominal pain, and diarrhea of watery loose stools. In serious cases, the patients show persistent high fever that rapidly progresses into obvious pneumonia, acute lung injury (ALI), acute respiratory distress syndrome (ARDS), pulmonary hemorrhage, pleural effusion, multiple organ failure, disseminated intravascular coagulation (DIC), shock, and Reye syndrome. Secondary bacterial infection may also occur to complicate the disease that progresses into septicemia, with a mortality of up to above $50 \%$.

\subsubsection{Radiological Demonstration}

Due to virus infiltration, the lung of patients with human infected H5N1 avian influenza is demonstrated with flakes of opacity that is predominantly exudates by chest X-ray or chest CT scan, namely the ground glass opacity and lung consolidation. In serious cases, the lesions in lungs progress rapidly, radiologically demonstrated as large ground glass opacity and lung consolidation. In the advanced stage, both lungs are shown with diffuse consolidation.

Human infected H5N1 avian influenza is radiologically characterized by:

1. Early increased density opacity and ground glass opacity in upper lung field

In the early stage after onset, the upper lung field is demonstrated with flakes of increased density opacity and ground glass opacity, which are the radiological signs of human infected avian influenza.

2. Diffuse lesion

The invasion of H5N1 avian influenza virus to human lung tissues causes an extensive range of lesions, with diffuse and exudative lesions in multiple lobes and multiple segments of both lungs. At the peak of their progression, most of lung fields in both lungs are involved, with large flakes of cloudy dense mass like opacity or even white-lung sign.

3. Rapid changes of the lesion

Radiologically, the lesions are demonstrated with rapid changes. A lesion that is initially found in one lung field may rapidly develop to involve the upper, middle and lower lung fields within $24-48 \mathrm{~h}$, radiologically demonstrated as involvement of the whole lung lobe.

4. Concurrently involved pulmonary parenchyma and interstitium

Due to necrosis and shedding of pulmonary alveoli, the pulmonary alveoli contain less gas but are filled with exudates such as various serous fluids, fibrin, erythrocytes, and neutrophilic granulocytes. In addition, hyaline membrane obviously forms in the alveolar space. Therefore, the lesions of human infected H5N1 avian influenza are radiologically demonstrated as alveolar exudates and lung consolidation, with the involved exudative lesions overlapping with pulmonary interstitium. After the exudative lesions are absorbed, grid like, flakes, strips and patches of opacity is shown, which resemble to the radiological signs of viral pneumonia.

5. Slow absorption of the lesion

The lesions are slowly absorbed. After the invasive assisting mechanical ventilation is retrieved with subsequent normal body temperature, breathing rate and WBC count, the both lungs are still radiologically demonstrated with strips, flakes, grid like, and patches of consolidation opacity, indicating inconsistency between chest radiology and clinical symptoms due to long-term progression of the lesions. That is to say, the absorption of lesions in lung lags behind clinical manifestations. In adult patients, the pleura is involved in different degrees, with a small quantity of pleural effusion isolateral to the primary lesion as well as bilateral pleural thickening and adhesion. During the absorption stage, multiple lesions of lobular paraseptal emphysema are revealed, with no obvious signs of hilar and mediastinal lymphadenectasis. 


\subsection{Typical Cases}

\section{Case 1}

\section{[Brief Medical History]}

A 31-year-old male truck driver experienced fever, cough and shortness of breath since Jun. 3, 2006. After he paid a clinic visit in a local hospital, a diagnosis was made to be common cold and he received corresponding therapy. At 0:30 a.m. on Jun. 10, 2006, he was hospitalized due to aggravated condition. By physical examination, the body temperature was $39.2-40{ }^{\circ} \mathrm{C}$, heart rate $127 / \mathrm{min}$, breathing rate $32 / \mathrm{min}$ and blood pressure 116/80 mmHg. He showed a typical development, moderate nutrition, active position and slight unconsciousness. In addition, he showed eye conjunctival congestion and edema, identically sized and round pupils and obvious cyanosis in oral lips. By percussion, the left lung flatness; by auscultation, coarse breathing sound of both lungs that attenuated at the right lung. At d 15 prior to the onset, his wife ever went to buy an alive chicken at a market and brought it back home after having it killed on site. His wife and daughter showed no signs of pneumonia. By routine blood test, WBC $4.20 \times 10^{9} / \mathrm{L}, \mathrm{N} 0.75$, L 0.24 , HGB $127 \mathrm{~g} / \mathrm{L}, \quad$ RBC $4.84 \times 10^{12} / \mathrm{L}, \quad$ PLT $132 \times 10^{9} / \mathrm{L}$. By blood glass analysis, $\mathrm{pH} 7.426, \mathrm{PaO}_{2}$ $63 \mathrm{mmHg}, \mathrm{PaCO}_{2} 34 \mathrm{mmHg}, \mathrm{SO}_{2} \% 92 \%$. By blood biochemistry, GLU $7.87 \mathrm{mmol} / \mathrm{L}$, BUN $2.8 \mathrm{mmol} / \mathrm{L}, \mathrm{Cr}$ $88 \mu \mathrm{mol} / \mathrm{L}$, ALT $30 \mathrm{U} / \mathrm{L}$, and AST $66 \mathrm{U} / \mathrm{L}$. T cell count in peripheral blood was $5 / \mu 1$. Etiologically, RT-PCR by QIAGEN One Step RT-PCR kit was performed to examine his tracheal secretions. The examination report indicated that the target segments of $\mathrm{H} 5$ and $\mathrm{N} 1$ were amplified. Throat swab for RNA of H5N1 (PCR) twice were positive. All the above findings indicated the existence of H5N1 avian influenza virus.

[Radiological demonstration] See Fig. 10.1

[Diagnosis] Human infected H5N1 avian influenza complicated by pneumonia.

\section{[Discussion]}

Following the first case of human infected H5N1 avian influenza reported in HongKong in 1997, 6 cases of death were subsequently reported. By Dec. 31, 2014, a total of 40 cases was reported in China, with 27 cases of death, and the mortality was up to $67 \%$. The high death rate indicated strong virulence of avian influenza virus $\mathrm{H} 5 \mathrm{~N} 1$ and the rapid progression of the disease. Chest radiological examinations are of great importance for our understandings about its radiological demonstrations, which facilitate its diagnosis, differential diagnosis and treatment.
Via systematic examination as well as dynamic observation and analysis, the radiological demonstrations and all the dynamic changes of the lesions were harvest from the patient. The radiological demonstrations are characterized by:

1. On d 7 after onset, the first chest X-ray demonstrated large flakes of increased density opacity in the left upper and middle lung fields. On d 8 after onset, the first chest CT scan demonstrated large consolidation opacity with high density in the apico-posterior and anterior segments of the left lung and in the left lower lung lobe, with air bronchogram inside. The anterior segment of the right upper lung was shown with ground glass opacity. All the findings indicated that ground glass opacity and large consolidation opacity are early signs of human infected avian influenza by chest radiology.

2. The invasion of human lung tissues by avian influenza virus $\mathrm{H} 5 \mathrm{~N} 1$ is extensive, demonstrated as diffuse and exudates lesions in both lungs with multiple segments and lobes involved. At the peak of its progression, most of the lung fields in both lungs is involved, demonstrated as large cloudy dense mass like opacity and even white-lung sign.

3. Radiologically, the lesions change rapidly. The initial lesions in the left upper and middle lung fields can extend into the left lower lung field within $24 \mathrm{~h}$ to involve the whole lung. In the following $48 \mathrm{~h}$, the lesions may rapidly extend into the right upper, middle and lower lung fields to involve the whole lungs.

4. The lesions are absorbed slowly. In this case, on d 35 after onset, the invasive assisting mechanical ventilation was retrieved, and the patient subsequently showed normal body temperature, breathing rate and WBC count. On d 33 after onset, chest CT scan still showed strips, flakes, grid like and patches of consolidation opacity with obvious air bronchogram inside. The follow-up CT scan was then performed each year, and the 5th chest CT scan showed interstitial fibrosis in both lungs, demonstrated as lobular septal thickening, sub-pleural arc shape linear opacity, paraseptal emphysema, ground glass opacity, bronchoectasis and small consolidation opacity.

5. The pleura is involved in different degrees. Pleural effusion in a small quantity was demonstrated isolateral to the primary lesions, and bilateral pleura were shown to be thickened with adhesion. 

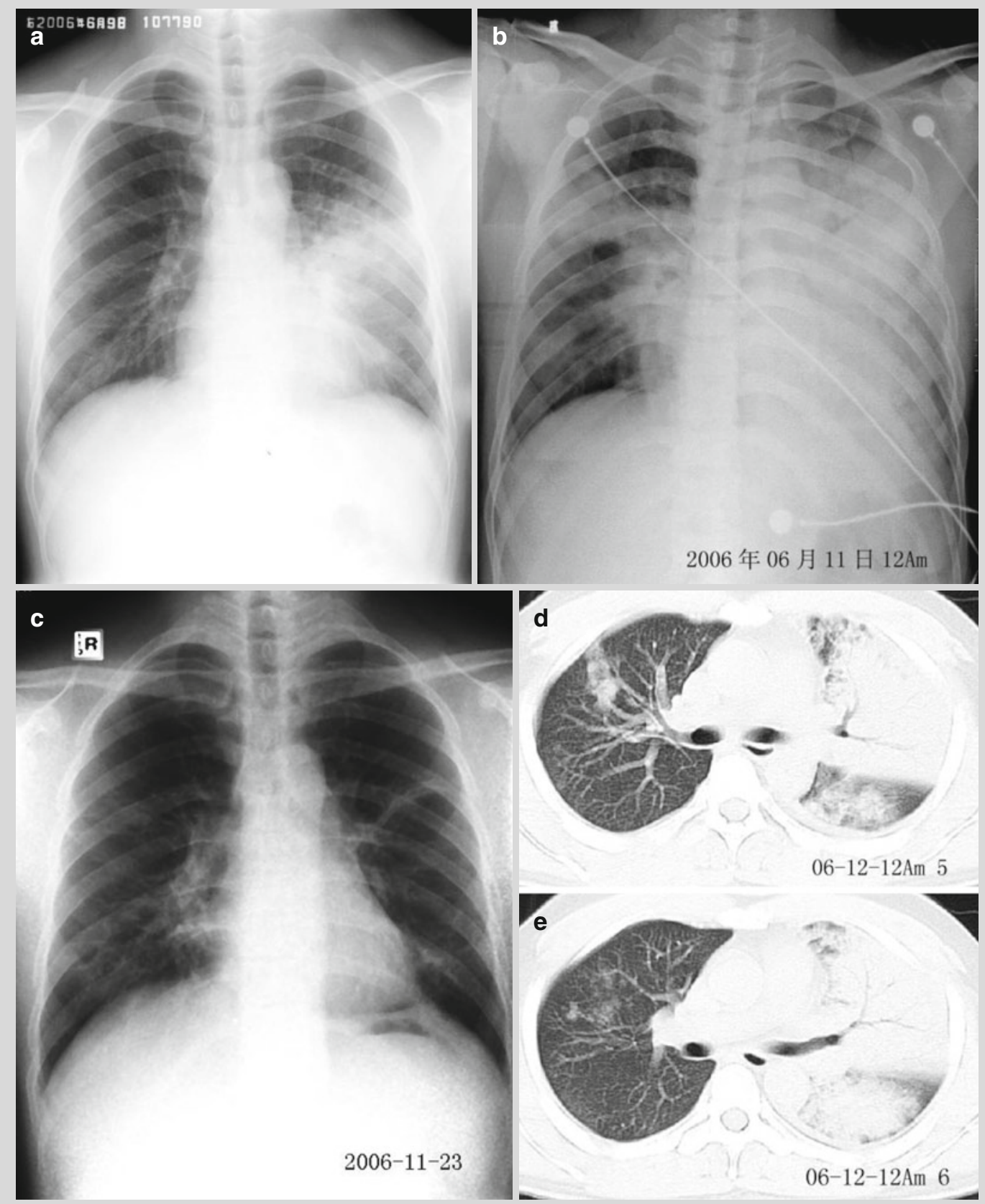

Fig. 10.1 (a) On d 6 after onset, chest X-ray demonstrated poorly defined large flakes of increased density opacity at the left middle and lower lung fields, and some of the lesions showed ground glass opacity. (b) On d 8 after onset, chest X-ray reexamination demonstrated rapid progression of the lesions, with large flakes of increased density opacity at the whole left lung fields in white-lung sign. Flakes of increased density opacity were revealed at the right hilum area of right middle lung field and middle-medial zone of right lower lung field. (c) On d 20 after onset, chest X-ray reexamination demonstrated sporadic spots and cords like increased density opacity in both lungs, with well defined boundaries. (d, e) On d 7 after onset, CT scan showed light small flakes of opacity at the posterior segment of right upper lung lobe and medial segment of the right middle lung lobe. Irregular small flakes of consolidation was revealed at the apico-posterior segment and anterior segment of left upper lung lobe. Large flake of dense opacity of consolidation was also revealed at the lingual segment of the upper lung lobes and most of the lower lung lobes, with air brochogram inside. Left pleural effusion was also demonstrated in a moderate quantity. (f, g) On d 34 after onset, CT scan showed ground glass opacity at the apical and posterior segments of right upper lung lobe, patches of consolidation at the anterior segment of right upper lung lobe with air bronchogram inside, comparatively shrinkage of the right middle lung lobe in dense opacity of consolidation with air bronchogram inside. The right lower lobe was revealed with scattering irregular patches of opacity, fine arch like linear opacity dorsally near the pleura, and slight thickening of the adjacent pleura. The left upper lung lobe was demonstrated with generally increased density opacity with air bronchogram inside and surrounding lobular septal thickening that reaches subpleura. The lower lung lobe showed slightly small volume, with aggregating lung markings, extensive consolidation and thick air bronchogram inside. (h, i) On d 286 after onset, CT scan revealed the lesions in lungs to be pulmonary interstitial changes including fibrous cords like, grid like and ground glass opacity 

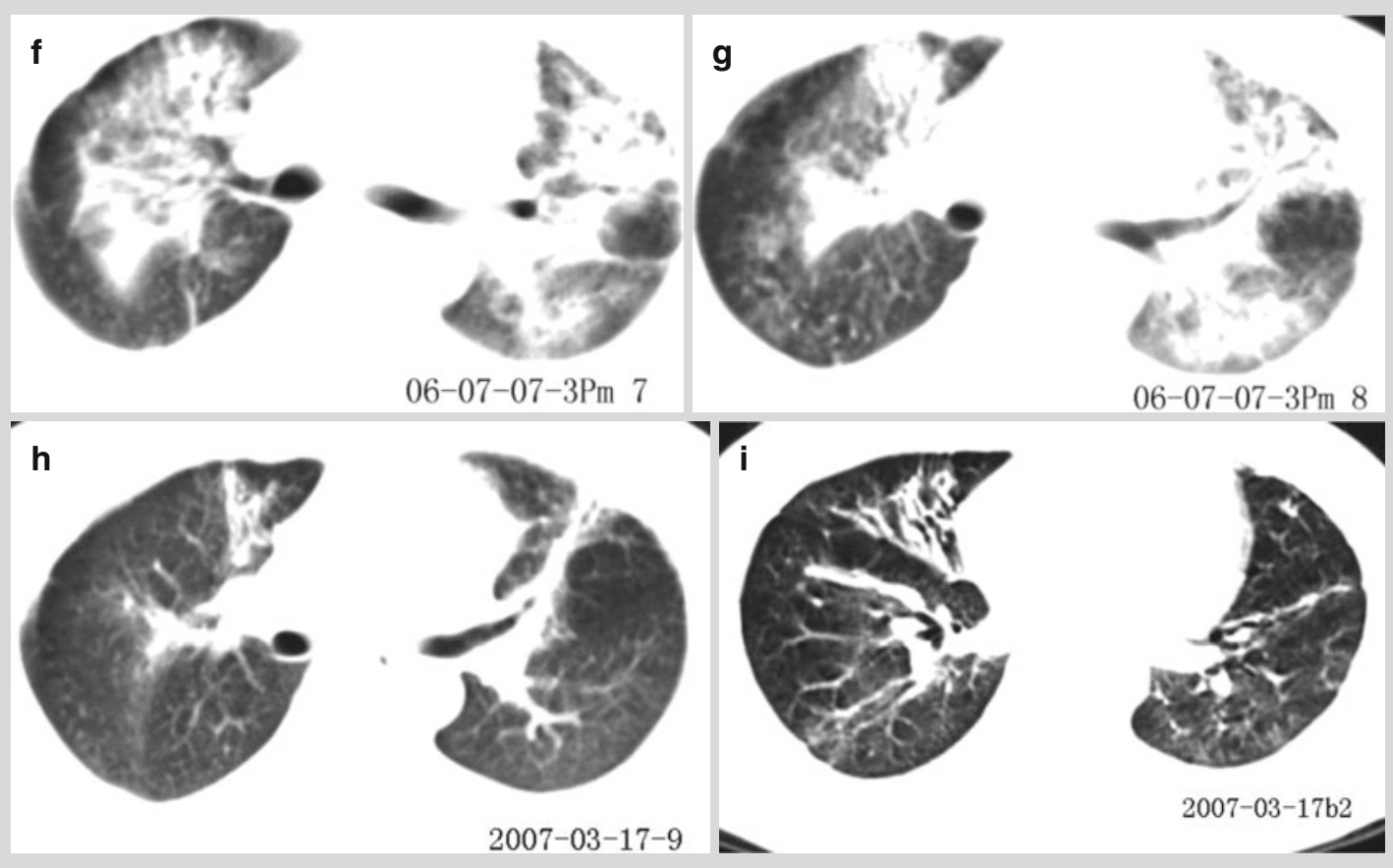

Fig. 10.1 (continued)

The occurrence complications following human infected avian influenza is the direct cause of death and should be highly concerned in clinical practice. The common complications and their prognoses are described as the following:

\section{Acute respiratory distress syndrome (ARDS)}

In the most serious stage of the disease, extensive consolidation opacity in both lungs, namely the whitelung sign, indicates the occurrence of ARDS. In this cases, the patient showed the sign of ARDS on d 9 after onset. After invasive mechanical positive pressure ventilation and anti-anoxia therapy were administered, his condition was improved. And multiple organs dysfunction syndrome (MODS) was controlled.

2. Pulmonary secondary infection

Common pulmonary secondary infections include pseudomonas aeruginosa infection, mycotic infection and other bacterial infections. Since d 10 after onset, the patient was detected for several times with growth of pseudomonas aeruginosa in his phlegm specimens. Particularly on d 20 after onset, his WBC count obviously increased and chest X-ray demonstrated patches uneven opacity in the left lower lung. After polymyxin $\mathrm{B}$ and other medications were administered, his pulmonary secondary infection was controlled.

3. Pulmonary fibrosis

Although his condition was stable with normal vital signs, on discharge his both lungs were shown with irregular grid like, fibrous strips and patches of opacity and accompanying local pleural thickening by thin layer CT scan. These findings indicated existence of pulmonary fibrosis, whose underlying mechanism may be hyperplasia of fibrous tissues and capillaries caused by fibrosis and organization of exudates as well as formation of microthrombosis in pulmonary capillaries based on formation of alveolar hyaline membrane and fibrin exudation. However, the hypothesis needs to be proved by sufficient evidence and the dynamic changes of pulmonary fibrosis still need to be further studied.

4. Pleural effusion

In this case, the patient showed left pleural effusion in a small quantity on $\mathrm{d} 8$ after onset by the first chest CT scan. On d 33 after onset, the second chest CT scan demonstrated absorption of pleural effusion as well as local pleural thickening and adhesion. These findings indicated pleura is involved in patients with human infected avian influenza, which has been further proved by autopsy.

H5N1 avian influenza should be differentiated from the following diseases.

\section{Severe acute respiratory syndrome (SARS)}

The lung changes of both human infected avian influenza and SARS are mainly parenchymal and interstitial lesions, with similar shape, development and outcome. Therefore, their differential diagnosis is challenging, and 
depends mainly on etiological examination and epidemiological history.

SARS is an acute respiratory infectious disease caused by novel corona virus. At its early stage, radiology demonstrates pulmonary interstitial changes, predominantly ground glass opacity. During its progressive stage, the lesions are demonstrated as predominantly ground glass opacity and rarely large consolidation opacity. The lung lesions of human infected H5N1 avian influenza show rapid changes. In some serious patients, the lesions in lungs may change within several days or even 1 day, with rapid progression from small area to large area, from upper or lower lung to the whole lung, from one lung to both lungs, and from ground glass opacity to consolidation opacity. However, in patients with SARS, the lesions in lungs progress slowly, and some lesions are migratory.

The lesions of human infected H5N1 avian influenza are in one lung or both lungs, possibly in upper and lower lung. In serious cases, the lesions are diffuse in both lungs, with more serious condition at the lower lungs. However, in the early stage of SARS, the nodular lesions are commonly located in the subpleural lateral lung field.

\section{Type A viral pneumonia}

Based only on radiological findings, pneumonia induced by H5N1, H1N1 and H7N9 avian influenza viruses can hardly be differentiated. And their differential diagnosis is mainly based on epidemiological history and etiological examination.
Pneumonia induced by H1N1, H5N1 and H7N9 avian influenza virus is caused by influenza A virus, with clinical influenza like manifestations. Chest CT scan demonstrates the diseases with ground glass opacity and consolidation opacity of varying sizes. Compared to pneumonia induced by $\mathrm{H} 1 \mathrm{~N} 1$ avian influenza virus, the lesions of pneumonia induced by $\mathrm{H} 5 \mathrm{~N} 1$ and $\mathrm{H} 7 \mathrm{~N} 9$ avian influenza virus are radiologically demonstrated with a larger range, with more rapid progression and more common air bronchogram. The lesions of pneumonia induced by $\mathrm{H} 5 \mathrm{~N} 1$ avian influenza virus show the more rapid progression, followed by H7N9 and then H1N1.

By chest $\mathrm{CT}$ scan, pneumonia induced by $\mathrm{H} 5 \mathrm{~N} 1$ avian influenza virus is demonstrated with large ground glass opacity and consolidation opacity in both lungs that distribute extensively and progress rapidly. In some cases, the lesions are migratory, with slow absorption and obvious pulmonary interstitial fibrosis. Its mortality rate is around $60 \%$. Pneumonia induced by H7N9 avian influenza virus is radiologically demonstrated with initial lesions in bilateral middle and lower lungs that are predominantly ground glass opacity and consolidation opacity. These lesions show rapid progression and relatively slow absorption. And its mortality rate is about $36 \%$. Pneumonia induced by H1N1 avian influenza virus is radiologically demonstrated as multiple flakes of ground glass opacity as well as patches and large flakes of high density consolidation, possibly with lobar or segmental atelectasis and pleural effusion. And its mortality rate is about $6 \%$. 


\section{Case 2}

\section{[Brief Medical History]}

A 26-year-old woman, being pregnant for more than 2 months, reported a history of fever since Feb. 11, 2006, with a body temperature of above $38.5^{\circ} \mathrm{C}$ and the highest body temperature of up to $40{ }^{\circ} \mathrm{C}$. She also experienced cough with a little phlegm as well as diarrhea early after onset. In a local hospital, routine blood test showed WBC count $6.1 \times 10^{9} / \mathrm{L}, \mathrm{GR} \% 74.9 \%$ and LY\% $15.6 \%$. Chest $\mathrm{X}$-ray indicated pneumonia at the right lower lung. By physical examination, breathing sound at the right lower lung was low and no other obvious positive signs. On d 7 after onset, Feb. 18, 2006, she was transferred to another hospital due to persistent fever, aggravated cough with more phlegm, chest distress and difficulty breathing. By inquiries of her epidemiological history, she reported a definitive history of contact to sick/dead chicken in her home 7 days prior to the onset, Feb. 4, 2006. By routine blood test, WBC count $3.30 \times 10^{9} / \mathrm{L}, \mathrm{N} 0.83$, L 0.15 and PLT $56 \times 10^{9} / \mathrm{L}$. Blood biochemistry showed AST 90U/L, CK 74U/L, and LDH 537U/L. By blood gas analysis, pH 7.426, $\mathrm{PaO}_{2} 25.1 \mathrm{mmHg}, \mathrm{PaCO}_{2} 51 \mathrm{mmHg}, \mathrm{HCO}_{3}$ $19.4 \mathrm{mmol} /$, and $\mathrm{SO}_{2} \% 92 \%$. Respiratory secretions examination on $\mathrm{d} 9$ after onset (specimens collected 1 day ago), Feb. 20, 2006, the virus nucleic acid positive for $\mathrm{H} 5 \mathrm{~N} 1$ avian influenza virus. By reexamination by national parallel laboratory, the finding was still positive, with H5N1 avian influenza virus isolation positive.

The patient experienced dyspnea and rapid progression on $\mathrm{d} 7$ after onset, and the condition further progressed into ARDS on $\mathrm{d} 8$ after onset. Because the simplex oxygen supplying therapy failed to maintain oxygenation, noninvasive mechanical ventilation was ordered on $\mathrm{d} 9$ after onset to maintain continuous positive airway pressure (CPAP) with settings of $10 \mathrm{~cm} \mathrm{H}_{2} \mathrm{O}, \mathrm{FiO}_{2} 45 \%$, and an elevated $\mathrm{SaO}_{2}$ to $90-93 \%$. But the noninvasive mechanical ventilation was discontinued due to shortness of breath and frequent cough of the patient. Subsequently, invasive nasotracheal intubation for ventilation was performed on that day. Tracheotomy was then performed for invasive ventilation 3 days after nasotracheal intubation.

On d 10 after onset, her body temperature returned to normal, but on d 14 after onset, the body temperature rose again to $37.5{ }^{\circ} \mathrm{C}$, with increased peripheral hemogram and yellowish purulent bronchial secretions. A diagnosis of secondary bacterial infection was made and the administered antibiotics were adjusted accordingly. On d 16 after onset, miscarriage occurred. And on d 18 after onset, the invasive ventilation was discontinued, which was given for 10 days. On d 21 after onset, tracheal extubation was performed. And on d 20 after onset, her hemogram returned to normal indicating controlled secondary infection and convalescent stage of the disease. On Mar. 23, 2006 ( 40 after onset), the patient was discharged according to the criteria in Clinical Guidelines for Human Infected Avian Influenza (revised version, 2005) formulated by Ministry of Health, P. R. China.

[Radiological demonstration] Fig. 10.2.

[Diagnosis] Human infected H5N1 avian influenza.

\section{[Discussion]}

The patient is characterized by young woman, contact to sick/dead chicken 7 days before onset, initial symptom of fever, and normal WBC count. Her condition rapidly developed into pneumonia within 1 week after onset, and further rapidly into respiratory failure and multiple organs dysfunction.

Chest X-ray showed early exudation opacity in the right lower lung ( 4 after onset), and exudation opacity in bilateral middle and lower lung fields (d 6 after onset). On d 11 after onset, chest X-ray showed the most serious conditions, with large consolidation opacity in the right lung and the left middle lung. On d 12 after onset, oxygenation was improved, but slow absorption of the lesions was revealed by both chest X-ray and chest CT scan. On discharge, chest CT scan still demonstrated extensive spots, flakes and cords like opacity in both lungs.

The case is special in the following aspects:

\section{Rapid progression of the condition.}

On d 6 after onset, chest X-ray demonstrated obvious deterioration of lesions every $3 \mathrm{~h}$. Clinically, the patient experienced dyspnea and blood gas analysis indicated respiratory failure.

2. Pregnancy.

At the onset of the symptoms, the patient was pregnant for more than 2 months and miscarriage occurred on $\mathrm{d} 16$ after onset, which was within the expectation of the physicians. Complete curettage of uterine cavity following intraveneous anesthesia was successfully performed to remove fetus and placenta.

3. Blood gas analysis and chest X-ray.

On d 12 after onset, blood gas analysis, routine blood test and biochemical examination indicated improved condition of the patient. And on d 20 after onset, the above examinations indicated normal condition of the patient. However, chest X-ray and chest CT scan still demonstrated slow absorption of the consolidation opacity. On discharge, blood gas analysis indicated normal but chest CT scan demonstrated obvious pulmonary interstitial changes.

This case is one of the cured cases of human infected avian influenza, with comparatively severe condition and 

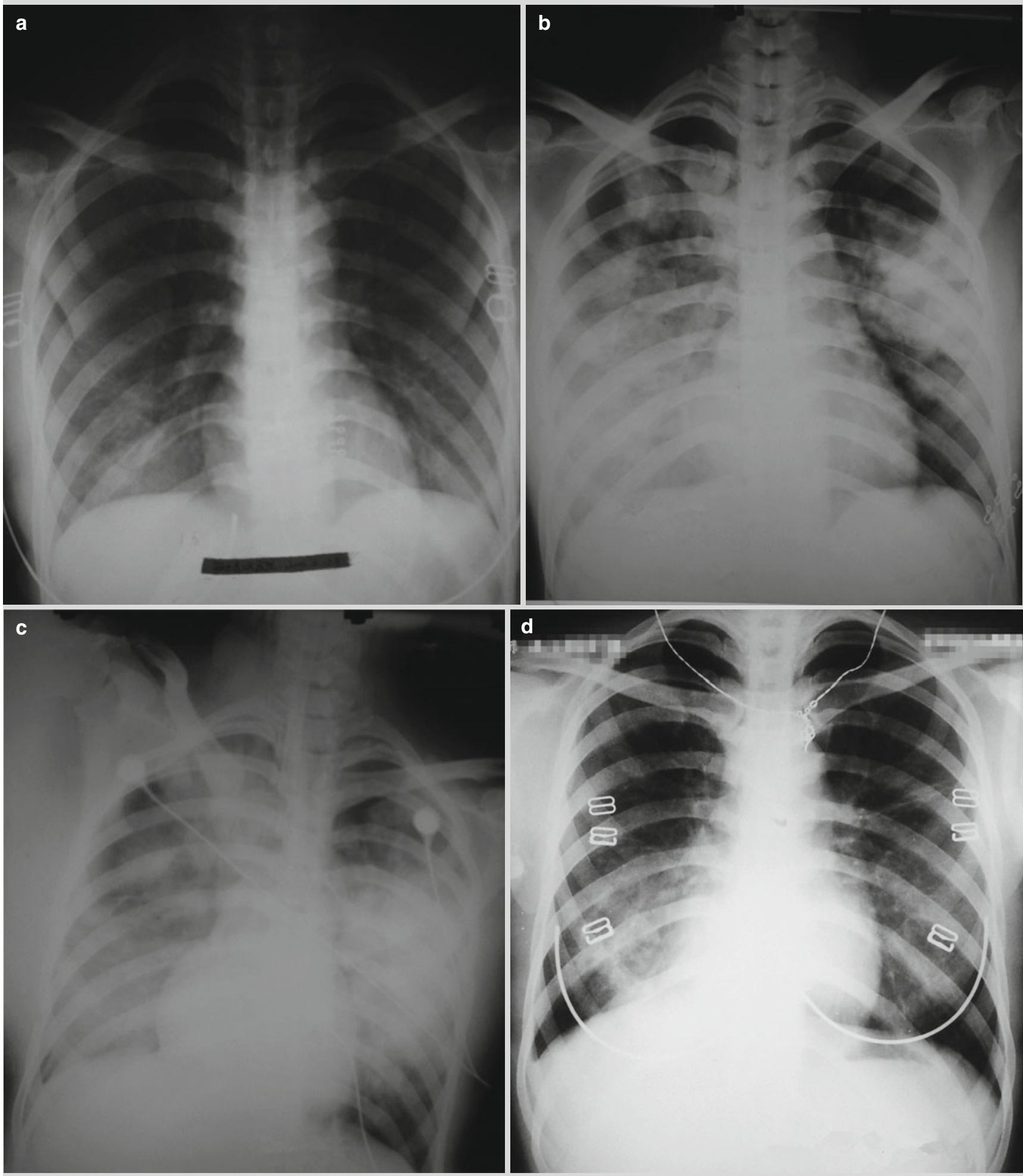

Fig. 10.2 (a) On d 4 after onset, chest X-ray demonstrated poorly defined flake of light opacity in the right lower lung field, and well defined left lung. (b) On d 7 after onset, the lesions were shown with rapid progression in a larger range in both lungs. Both lungs were shown with extensive cloudy and cotton like opacity and fusion of some into large flake. (c) On d 11 after onset, the lesions were shown to be within an even larger range in both lungs, namely the white-lung sign in both lungs, which indicated relapse and progression of the lesions. (d)
On d 72 after onset, the both lungs were demonstrated with interstitial lesions, characterized by grid like opacity and obviously slower absorption. (e-g) On d 7 after onset, CT scan demonstrated extensive distribution of lesions in both lungs, which were more serious at the dorsal parts of both lungs, with air bronchogram in consolidation opacity. (hj) On d 72 after onset, CT scan demonstrated obvious absorption of the lesions, cords like opacity in ground glass like change, and interlobular septal thickening in the peripheral areas of both lungs 


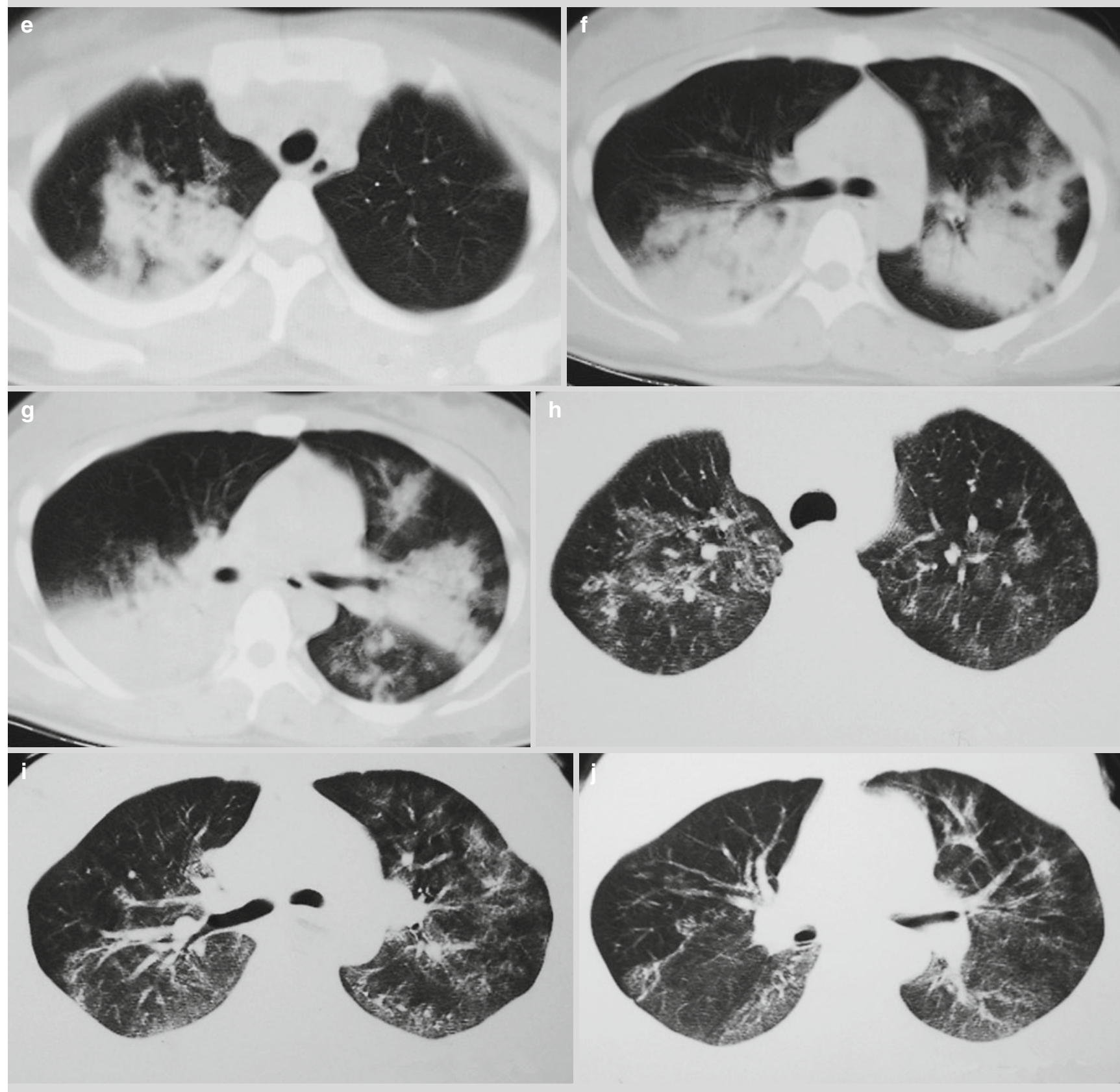

Fig.10.2 (continued)

less complications. Only on d 14 after onset, the patient experienced re-elevated body temperature to $37.5^{\circ} \mathrm{C}$, with elevated peripheral hemogram and yellowish purulent bronchial secretions. The condition was diagnosed as secondary bacterial infection and the administered antibiotics were adjusted accordingly. On d 20 after onset, the hemogram returned to normal, indicating controlled secondary infection and convalescent stage of the disease. After her discharge, her serum collected at the convalescent stage was collaboratively administered to cure a patient with human infected H5N1 avian influenza in Shenzhen, Guangdong, China, which is known as the patient with the most serious condition among patients with $\mathrm{H} 5 \mathrm{~N} 1$ avian influenza.

By radiology, the key points for differential diagnosis are as the following:

Based on early chest CT scan, the lesions are demonstrated as poorly defined large flakes of increased density opacity, which are bilaterally symmetric. In combination to the clinical symptoms of high fever and cough, it 
should be differentiated from bacterial pneumonia of both lungs. However, the patient reported a history of contact, with normal level of peripheral WBC count. By dynamic observations via radiological examination, the lesions in both lungs progressed rapidly, with extensive involvement in bilateral lung fields and concurrent ground glass opacity and pulmonary consolidation opacity. All of these manifestations and findings are in consistency with pneu- monia induced by human infected avian influenza. In addition, pneumonia induced by highly pathogenic H5N1 avian influenza virus should also be differentiated from SARS, AIDS complicated by PCP, and pneumonia induced by H7N9 avian influenza virus. Further differentiation should be comprehensively based on epidemiological history, etiological examination, clinical manifestations, and radiological findings.

\section{References}

Lu PX, Zhou BP. Clinical imaging diagnosis of emerging infectious diseases. Beijing: People's Medical Publishing House; 2013.

Lu PX, Zhou BP, Zhu WK, et al. Radiological demonstrations of pneumonia induced by highly pathogenic $\mathrm{H} 5 \mathrm{~N} 1$ avian influenza virus. Chin J Med Imaging Technol. 2007a;23(4):532-5.

Lu PX, Zhu WK, Ye RX, et al. CT demonstrations and their dynamic changes in adult patients with severe pneumonia induced by H5N1 avian influenza virus. Chin J CT MRI. 2007b;5(1):31-4.

Lu PX, Wang PX, Zhou BP, et al. Radiological features of lung changes caused by avian influenza subtype A H5N1 virus: report of two severe adult cases with regular follow-up. Chin Med J (Engl). 2010;123(1):100-4.

Zhou BP, Li YM, Lu PX. Human infected avian influenza. Beijing: Science Press; 2007. 